\title{
THE
}

\section{Quantum Spin Chains and the Conformal Anomaly}

Jill C. Bonner

University of Rhode Island

John B. Parkinson

Follow this and additional works at: https://digitalcommons.uri.edu/phys_facpubs

Terms of Use

All rights reserved under copyright.

\section{Citation/Publisher Attribution}

Jill C. Bonner and John B. Parkinson. Quantum spin chains and the conformal anomaly. J. Appl. Phys. 63 (1988), 3543-3545.

Available at http://dx.doi.org/10.1063/1.340737.

This Article is brought to you for free and open access by the Physics at DigitalCommons@URI. It has been accepted for inclusion in Physics Faculty Publications by an authorized administrator of DigitalCommons@URI. For more information, please contact digitalcommons-group@uri.edu. 


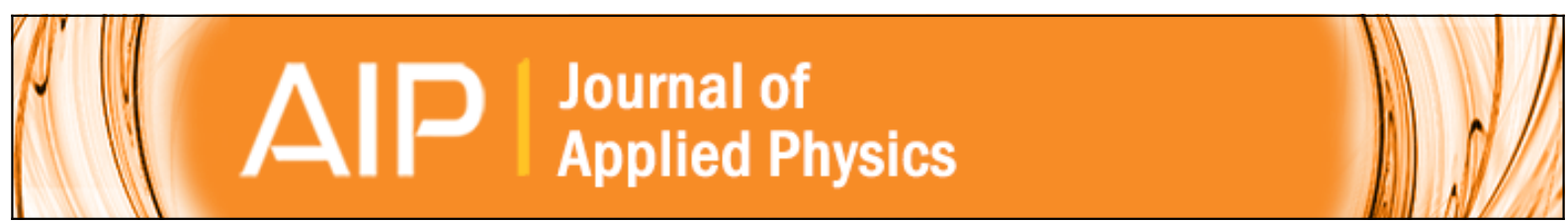

\section{Quantum spin chains and the conformal anomaly}

Jill C. Bonner and John B. Parkinson

Citation: Journal of Applied Physics 63, 3543 (1988); doi: 10.1063/1.340737

View online: http://dx.doi.org/10.1063/1.340737

View Table of Contents: http://scitation.aip.org/content/aip/journal/jap/63/8?ver=pdfcov

Published by the AIP Publishing

\section{Articles you may be interested in}

NMR relaxation rate of a quantum spin chain with an impurity

Low Temp. Phys. 38, 639 (2012); 10.1063/1.4730977

Nonintegrability and quantum spin chains

J. Appl. Phys. 61, 3950 (1987); 10.1063/1.338594

Generalized Heisenberg quantum spin chains (invited)

J. Appl. Phys. 61, 3941 (1987); 10.1063/1.338592

Anisotropic quantum spin chains

J. Appl. Phys. 52, 1968 (1981); 10.1063/1.329586

Heisenberg chains with alternating spin quantum numbers

J. Appl. Phys. 50, 7401 (1979); 10.1063/1.326907

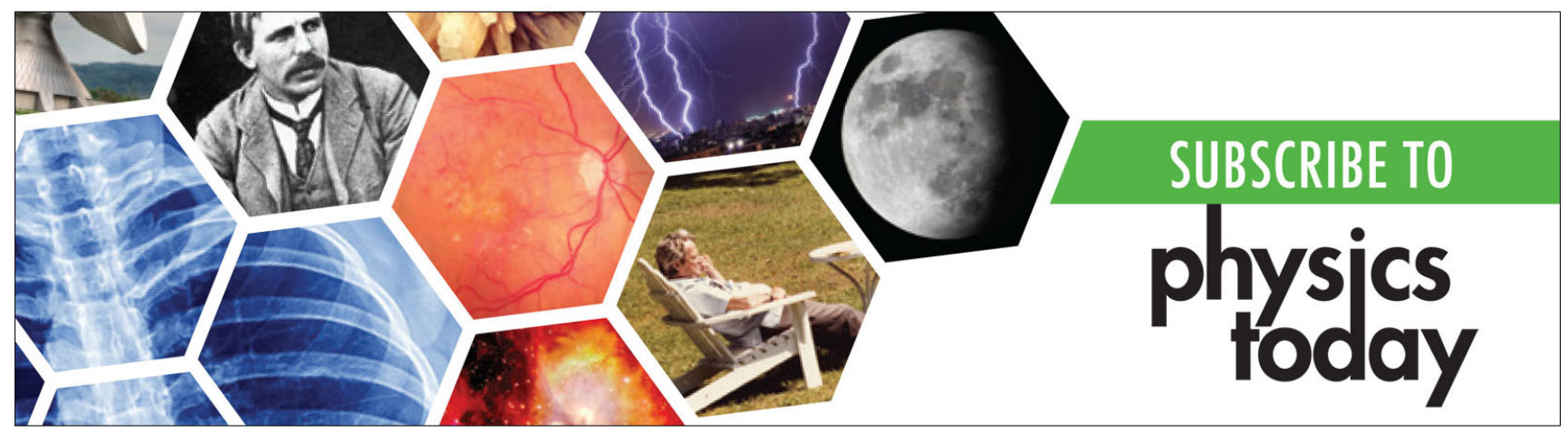




\title{
Quantum spin chains and the conformal anomaly
}

\author{
jill C. Bonner \\ Department of Physics, The University of Rhode Island, Kingstor, Rhode Island $02881-0817$ \\ John B. Parkinson \\ Department of Mathematics, University of Manchester Institure of Science and Technology (UMIST), \\ Manchester, M6O IQD, United Kingdom
}

\begin{abstract}
The conformal anomaly $c$ determines the universality class of a model system in statistical mechanics. The value of characterizes both 2D ciassical models and their $1 \mathrm{D}$ quantum counterparts. The conformal anomaly may therefore be determined numerically for quantum spin chains using the relation: $E_{0}(N) \approx E_{0}(\infty)-(N \Delta E / 12) c\left(1 / N^{2}\right)$, where $E_{0}(N)$ is the ground-state energy of an $N$-spin ninite system, $E_{0}(\infty)$ is the ground-state energy in the thermodynamic limit, and $\Delta E$ is the energy gap between the ground state at $k=0$ and the first excited state of the dispersion curve at $k=2 \pi / N$. The numerical approach is highly successful when tested on the integrable $s=\frac{1}{2}$ Heisenberg antiferromagnetic $X X Z$ chair and the integrable $s=1 \mathrm{SU}(2)$ model. The method gives $c=1$ to within $2 \%$ accuracy for the $s=1$ and $\frac{3}{2} X Y$ chains, placing them in the universality class of the $2 \mathrm{D} X Y$ model. The result $c=1$ ( $2 \%$ accuracy) is obtained for the $s=\frac{3}{2}$ Heisenberg antiferromagnetic chain, in agteement with the Haldane prediction. The $s=1$ pure antiferromagnetic biquadratic chain and the $s=1$ $X X Z$ model with uniaxial anisotropy in the vicinity of the critical point $\Delta=\Delta_{2} \sim 1.15-1.18$ have also been studied.
\end{abstract}

\section{INTROOUCTION}

Conformal invariance ${ }^{1,2}$ has provided a powerful tool for classifying statistical mechanical systems according to their critical behavior. A system at criticality is invariant with respect to a conformal algebra, ${ }^{3}$ and is characterized by a number, the conformal anomaly, or central charge $c$ that determines the universality class of the system. The same value of $c$ characterizes both 20 classical models and their $1 D$ quantum counterparts. Biöte, Cardy, and Nightingale, ${ }^{4}$ and Affeck ${ }^{5}$ have shown that the conformal anomaly can be found by studying the large- $N$ behavior of finite $N$ chains, according to the expression

$E_{0}(N)=E_{0}(\infty)-(\pi v / 6) c\left(1 / N^{2}\right)+$ (higher terms),

where $E_{0}(\infty)$ is the ground state energy per spin in the thermodynamic limit, $N \rightarrow \infty ; E_{0}(N)$ is the ground state energy per spin of a finite, $N$-spin chain; $v$ is the velccity of sound for the model (i.e., the initial slope of the $\Delta E$ vs $k$ dispersion curve). Expression (1) holds only for chains with periodic boundary conditions.

It is known that $c=\frac{1}{2}$ for the spin- $\frac{1}{2}$ transverse Ising chatin (universality class of the 2D Ising model) and $c=1$ for the spin- $-\frac{1}{2} X X Z$ chain with continuous symmetry (universality class of the $2 \mathrm{D}$ Baxter model) from planar anisotropy analytic resuits. ${ }^{4}$ Quantum spin chains with higherspin provide examples of models with $c>1$. For a class of Bethe Ansatz integrabie models with SU(2) symmetry, ${ }^{6,7}$ Affeck ${ }^{5}$ has predicted the following expression for $\varepsilon$ :

$$
c=3 s /(1+s) \text {. }
$$

Obviously, each member of the class is in a different universality class, where $c=1$ for $s=\frac{1}{2}, c=1.5$ for $s=1, c=1.8$ for $s=\frac{3}{2}$ etc.

It has been realized by several authors ${ }^{8-10}$ in addition to ourselves (see brief summary in Ref. 11) that Eq. (1) may be formulated in a way that allows calculation of a series of estimates, $c(N)$, for a sequence of chains of increasing $N$, and extrapolation to $N=\infty$ to obtain the value of $c$. This numerical approach will be discussed in detail in the next section.

Voynarovich and Eckle studied the leading and nextto-leading finite-size corrections to the ground and first excited states for the spin- $\frac{1}{2}$ anisotropic Heisenberg model in the critical region. Since this model is Bethe Ansatz integrable they condd obtain the analytic form of the corrections. The Hamiltonian has the form

$$
H=J \sum_{i=1}^{N}\left(s_{i}^{x} s_{i+1}^{x}+s_{i}^{y} s_{i+1}^{y}+\Delta s_{i}^{z} s_{i+1}^{z}\right)
$$

where $\Delta$ is an anisotropy parameter. They showed explicitly the presence of $\ln N$ factors in the correction terms and also why a naive finite-size scaling analysis on this system yields a critical point substantially in error. ${ }^{12,13}$

More ${ }^{10}$ and the present authors are particularly interested in the critical behavior of spin-3 Heisenberg antiferromagnetic chains (HBAFM's) in comparison with the spin- $\frac{1}{2}$ HB AFM. The Haldane prediction ${ }^{14}$ is that both systems should be in the same universality class and thereby possess the same set of critical exponents, for example, $c=1$ and $\eta=1$. Affeck, on the other kand, initially conjectured that the spin- $\frac{3}{2} \mathrm{HB}$ AFM should be in the universality class of the $s=\frac{3}{2}$ integrable SU(2) model, with $c=1.8$ and $\eta=0.6 .^{15}$

Essentially all workers in this area have tested the numerical approach on the spin- $\frac{1}{2} X X Z \mathrm{AFM}$ with continuous symmetry, given by Eq. (1). The expected value $c=1$ has been found to high numerical accuracy in the appropriate parameter range $0 \leqslant \Delta \leqslant 1$. In addition, we have studied the $s=1$ integrable SU(2) chains, ${ }^{6.7}$ again finding numerical 
results in excellent agreement with Afleck's predicted value.

Our interest in numerical estimation of $c$ has been broader than that of other authors. We have employed the technique in the following connections.

(a) We have analyzed quantum spin chains which are not integrable to determine from the $c$ value the universality classes of these models. The spin- $-\frac{3}{2}$ HB AFM noted above is one example. Others of interest are the $s=1$ and $s=\frac{3}{2} X Y$ models, believed to be in the universality class of the Gaussian, $2 \mathrm{D} X Y$, or Baxter models. In the case of the spin-1 $X X Z$ chain, the uniaxial critical point at $\Delta=\Delta_{2}$ is predicted to be in the universality class of the $2 \mathrm{D}$ Ising model. ${ }^{14}$

(b) A $c$ value can only be defined if the statistical mechanical model is critical, i.e., gapless. If the numerical analysis is performed on a system which has an excitation gap, the result is a $c(N)$ sequence which plunges to zero. Hence, in principle, this method can be used to determine whether or not a given system is gapless, a question of considerabie interest since the controversial advent of the Haldane conjecture. $^{12,14}$ Tested on the integrable $s=\frac{1}{2} X X Z \mathrm{AFM}$ with uniaxial anisotropy (discrete symmetry) and a gap, the approach appears to work. Consequently, we have examined the $s=1 X X Z$ model at $\Delta=1$ (HB AFM) and also for $\Delta$ values in the range 1.1-1.2, encompassing the critical point $\Delta_{2} \sim 1.18$. The $s=1$ chain with pure AFM biquadratic exchange is also interesting. Oimaa, Parkinson, and Bonner, ${ }^{16}$ and Blöte and Capel ${ }^{17}$ obtained numerical evidence for a gap, in agreement with theoretical arguments of Afleck, Kennedy, Lieb, and Tasaki. "Sólyom, however, has numerical evidence against the presence of an excitation gap in the biquadratic nodel. ${ }^{19}$

(c) The common assumption is that nonintegrable systems will display critical behavior analogous to their integrabie counterparts, with unique $T_{c}$, associated scaling behavior, and a clearly defined universality class. However, the dramatically different dynamical behavior of both types of system suggest the possibility of differences in the static (spectral) properties also. Some unusual spectral phenomena in nonintegrable systems have aiready been observed. ${ }^{20}$ Hence $c$-rumber calculations on nonintegrable systems are of particular interest.

\section{NUMERICAL APPROACH}

Neglecting higher terms, we may write (1) as

$$
N^{2}\left[\left(E_{0}(\infty)-E_{0}(N)\right]=(\pi v / 6) c\right.
$$

The left-hand side (LHS) is essentially the asymptotic slope of a graph of $E_{0}(N)$ plotted versus $\left(1 / N^{2}\right)$, the intercept giving $E_{0}(\infty)$, which is generally not known for a nonintegrable system. Assuming a gapless $\sin k$-like, dispersion curve, the slope as $k \rightarrow 0$ is $v$, and is obtained numerically as

$$
v=\lim _{N \rightarrow 0}(N \Delta E / 2 \pi),
$$

where $\Delta E$ is the energy gap to the first excitation at $k=2 \pi / N$, since the finite- $N$ excitations are distributed across the Brillouin zone as multipies of $2 \pi / N$. Hence,

$$
(\pi v / 6)=\lim _{N \rightarrow \infty}(N \Delta E / 12)
$$

The value of $c$ can therefore be obtained in two ways.

(a) Extrapolate the slopes $s(N+2, N)$ of neighboring points on an $E_{0}(N)$ vs $N^{-2}$ plot, i.e., find

$$
\begin{aligned}
S & =\lim _{N \rightarrow \infty} s(N+2, N) \\
& =\lim _{N \rightarrow \infty} \frac{\left[E_{0}(N)-E_{0}(N+2)\right]}{\left[1 /(N+2)^{2}-1 / N^{2}\right]}
\end{aligned}
$$

Then

$$
c=\lim _{N \rightarrow \infty} \frac{s(N+2, N)}{N \Delta E / 12} .
$$

(b) Calculate finite- $N$ approximations to $c$ :

$$
c(N)=\frac{s(N+2, N)}{N \Delta E / 12}
$$

$$
\text { and then take limit } c=\lim _{N \rightarrow \infty} c(N) \text {. }
$$

Both approaches difier somewhat from the numerical approach of Moreo ${ }^{10}$ and seem to give more consistent results. Our calculations extend to rings of $N=14$ spins for $s=1$ and to $N=10$ spins for $s=\frac{3}{2}$.

\section{RESULTS}

$s=\frac{1}{2} A F M X X Z$ chain: As noted, for $0 \leqslant \Delta \leqslant 1$, the value $c=1$ is obtained to within $1 \%$ accuracy.

$s=1 S U(2)$ chain: Method (a) gives a value $c \sim 1.48$ and method (b) gives $c \sim 1.46$, in excellent agreement with the Affleck prediction $c=1.50$. The ground-state energy per spin $-E_{0}(\infty) \approx 4.000$.

$s=\frac{3}{2} H B$ AFM: Method (a) gives $c \sim 0.99$ and method (b) gives $c \sim 0.97$, in excellent agreement with the Haldane prediction $c=1$, and complete disagreement with the integrable $S U(2)$ predicted value of 1.8 . The ground-state energy per $\operatorname{spin}-E_{0}(\infty) \approx 5.080$.

$s=1$ XY: Method (a) gives $c \sim 0.98$ and method (b) gives $c-0.97$, strongly indicating the expected value $c=1.0$. The ground state energy per spin is $-E_{0}(\infty)$ $\approx 1.116$.

$s=\frac{3}{2} X Y:$ Method (a) gives $c-1.01$ and method (b) gives $c \sim 0.99$, strongly indicating the expected value $c=1.0$, as found also by Moreo. ${ }^{10}$ The ground-state energy per spin is $-E_{0}(\infty) \approx 2.4149$.

$s=1$ biquadratic AFM: This model is interesting since Refs. 16-18 predict a gap, albeit a small one, whereas Ref. 19 predicts no gap. Our extrapolations behave quite smoothly, with no indications up to $N=14$ spins of a sharp downward turn to zero, consistent with the presence of an excitation gap. Method (a) gives $c \sim 1.19$ and method (b) gives $c \sim 1.16$, consistent with a "round number" value $c=1.2$. This apparent $c$ value does not correspond to any known prediction for an $s=1 \mathrm{spin}$ chain. It is possible that the method is not sensitive to the presence of a very small gap until $N>14$, i.e., the asymptotic behavior of the $c(N) \mathrm{ex}-$ trapolations changes for $N>14$. By comparison, in the case 
of the nonintegrable spin- $\frac{3}{2} \mathrm{HB}$ AFM, however, all previous numerical studies have tended to indicate the presence of a gap, more like the case of spin-1 than spin $-\frac{1}{2}{ }^{21,22}$ Affeck and Lieb $^{23}$ have given a proof that all half-integer Heisenberg antiferromagnetic spin chains are gapless, but our $c$ calculation is essentially the first piece of numerical evidence of gapless behavior, predicted by Haidane. ${ }^{14}$

$s=1 X X Z$ chain, $\Delta>1$. We have studied anisotropy values $\Delta=1.1$ and 1.2 , encompassing the expected critical point $\Delta=\Delta_{2} \sim 1.15-1.18$. In both cases, the $(N)$ start from high values of $\sim 1.5$ and piunge downwards to zero, consistent with the presence of a gap. According to Haldane, at the critical point $\Delta_{2}$ the quantity $c \sim \lim _{N * \infty} c(N)=0.5$. We are "fine-scanning" the interval $1.1<\Delta<1.2$ to observe this phenomenon.

\section{CONCLUSION}

We have used a numerical approach involving finite chain calculations to determine the value of the conformal anomaly $c$ for a variety of spin chain models. Tested on the exactiy integrable $s=\frac{1}{2} X X Z$ chain with planar anisotropy and the $s=1$ integrable SU(2) model, our method gives excellent agreement with predicted values, which can also be obtained analytically. When applied to the $s=1$ and $s=\frac{3}{2}$ $X Y$ models, our method yields the value $c=1$ to within $2 \%$ accuracy, indicating that $X Y$ models of generai spin $s$ all beiong to the same universality class, that of the $2 \mathrm{D} X Y, 2 \mathrm{D}$ Gaussian, or Baxter model. When applied to the spin- $\frac{3}{2} \mathrm{HB}$ AFM, the method again yields the value $c=1$ to within about $2 \%$ accuracy, supporting the Haldane prediction that all half-integer spin HB AFM's are in the same universality class. The fact that the $s=1$ and $\frac{3}{2} X Y$ chains, and the $s=\frac{3}{2}$ HB AFM chain, comprising three models thought to be nonintegrable, all yield $c$ values as well-defined as those associated with integrable models, supports the belief that critical behavior is unaffected by nonintegrability.

The situation is at present less conclusive in the case of two additional models of interest. In the case of the $s=1$ chain with pure AFM biquadratic exchange, the existence of a gap is somewhat controversial, although strongly supported by theoretical arguments. We obtain an apparently well- defined $c$ value in the vicinity of 1.20 , instead of a trend to $c=0$ expected in the presence of a gap. In the case of the $s=1 X X Z$ model with uniaxial anisotropy, in the vicinity of the critical point $\Delta_{2} \simeq 1.15-1.18$, we hope to observe the val ue $c=\frac{1}{2}$ predicted by Haldane. Calculations at $\Delta=1.1$ and 1.2 indicate the presence of a gap, which is expected to vanish at, and only at, $\Delta=\Delta_{2}$. Further study of both models is indicated.

\section{ACKNOWLEOGMENTS}

We acknowledge M. P. Nightingale and $G$. Müller for reading the manuscript and for valuable comments. We are indebted to the National Science Foundation for Grant No. DMR86-03036 for financial support and to the Princeton Cyber 205 supercomputing system.

'A. M. Polyakov, JETP Lett. 12, 38 i (1970); D. Friedan, Z. Qiu, and S. Shenker, Phys. Rev. Lett. 56, 762 (1986).

2). L. Cardy, in Phase Transitions and Critical Phenomena, edited by C. Domb and J. Liebowitz (Academic, New York, 1986), Vol. 11, p. 55.

${ }^{3}$ M. A. Virasoro, Thys. Rev. D 10, 2933 (1970).

${ }^{4} \mathrm{H}$. W. J. Blöte, J. I. Cardy, and M. P. Nightingale, Phys. Rev. Lett. 56, 742 (1986).

51. Affeck, Phys. Rev. Lett. 56, 746 (1986).

LE. A. Takhtajan, Phys. Leti. 87A, 479 (1982).

${ }^{7}$ F. M. Babujian, Phys. Left. $90 \mathrm{~A}, 479(1982)$.

${ }^{8}$ F. C. Alcaraz, Research Report, The Australian National University (1986).

7F. Voynarovich and H.-P. Eckle, I. Phys. A 20, L97 (1987).

10. Morco (unpublished).

"H. W. J. Blöte and J. C. Bomer, Phys. Rev. 36, 2337 (1987).

123. C. Bonner and $G$. Muiler, Mys. Rev. B 29, 5216 (1984).

13. Sólyom and T. A. L. Ziman, Phys. Rev. B 30, 3980 (1984).

${ }^{14}$ F. D. M. Haldane, Phys. Lett. 93A, 464 (1983).

is . Afteck. Nucl. Phys. B 265, 409 (1986).

16J. Oitmaa, J. B. Parkinson, and J. C. Bonner, J. Phys. C 19, L95 (1986).

${ }^{17}$ H. W. J. Blöte and F. Capel, Physica A 139, 387 (1986).

${ }^{16}$ I. Afiteck, T. Kennedy, E. H. Lieb, and H. Tasaki, Phys. Rev. Lett. 59, 799 (1987).

19. Sólyom (unpublished).

${ }^{20}$ G. Müller, J. C. Bonner, and J. B. Parkinson, J. Appl. Phys. 61, 3950 (1987).

${ }^{21}$ J. B. Parkinson and J. C. Bonner, Phys. Rev. B 32, 4703 (1985).

22J. C. Bonner, G. Müller, M. P. Nightingale, H. W. J. Blöte, and J. B. Parkinson, J. Magn. Magn. Mater. 5457, 1253 (1986).

${ }^{23}$. Affeck and E. H. Lieb, Lett. Math. Phys. 12, 57 (1986). 